Article

\title{
A Buoyant, Microstructured Polymer Substrate for Photocatalytic Degradation Applications
}

\author{
John R. Bertram ${ }^{1,2}$ and Matthew J. Nee ${ }^{1, *}$ \\ 1 Department of Chemistry, Western Kentucky University, 1906 College Heights Blvd., \\ Bowling Green, KY 42101, USA \\ 2 Renewable and Sustainable Energy Institute, Materials Science and Engineering Program, University of \\ Colorado, Boulder, CO 80303, USA; jobe3667@colorado.edu \\ * Correspondence: matthew.nee@wku.edu; Tel.: +1-270-745-0114
}

Received: 25 September 2018; Accepted: 19 October 2018; Published: 22 October 2018

check for updates

\begin{abstract}
Microbubble fabrication of poly(dimethylsiloxane) (PDMS) beads with incorporated $\mathrm{TiO}_{2}$ provides a low-density, microstructured photocatalyst that is buoyant in water. This approach surmounts many of the challenges traditionally encountered in the generation of buoyant photocatalysts, an area which is critical for the implementation of widespread environmental cleaning of organic pollutants in water resources. Because the incorporation into the polymer bead surface is done at low temperatures, the crystal structure of $\mathrm{TiO}_{2}$ is unaltered, ensuring high-quality photocatalytic activity, while PDMS is well-established as biocompatible, temperature stable, and simple to produce. The photocatalyst is shown to degrade methylene blue faster than other buoyant, $\mathrm{TiO}_{2}$-based photocatalysts, and only an order of magnitude less than direct suspension of an equivalent amount of photocatalyst in solution, even though the photocatalyst is only present at the surface of the solution. The reusability of the $\mathrm{TiO}_{2} / \mathrm{PDMS}$ beads is also strong, showing no depreciation in photocatalytic activity after five consecutive degradation trials.
\end{abstract}

Keywords: photocatalytic degradation; inorganic-polymer hybrid; buoyant photocatalyst

\section{Introduction}

Photocatalytic degradation of organic pollutants has made significant progress since its early description by Tanaka and coworkers in 1985 [1]. Efforts since then have focused on the efficacy of the approach on various classes of compounds, including the mechanisms by which they proceed [2-6], and on generating photocatalysts that can be effective in different situations [7-10]. Although much progress has been made in developing compounds with improved photocatalytic activities, $\mathrm{TiO}_{2}$ remains a popular option for many applications because of its low-cost and environmental inertness [11-14]. For example, many materials have recently been functionalized by attaching $\mathrm{TiO}_{2}$ onto a support material for self-cleaning surfaces [15], microfluidic reactors [12], environmental remediation [16,17], etc. These materials include floating photocatalysts, in which $\mathrm{TiO}_{2}$ is attached to a buoyant support. There have been some reports of floating aerogel photocatalysts [18,19], but the most common method is to attach a photocatalyst to the surface of a buoyant support material. Supports in various forms have been reported in the literature, as reviewed by Singh et al. [20], but polymers are the most promising because of their chemical and environmental inertness, low-cost, and resistance to UV and oxidative processes. Polymer supports in the form of polyurethane foam [21], polystyrene sheets [22,23], and polypropylene granules [24] have been reported in the literature. However, in direct application to natural bodies of water, these support morphologies inhibit oxygen uptake and may not spread well over a large surface of contaminated water. Instead, the discrete bead morphology we use here is ideal in this situation because they can rotate on the surface of the water (maximizing exposure of the anchored photocatalyst), they are easy to ship and store, and their recovery from aqueous bodies is easily achievable. 
Poly(dimethylsiloxane) (PDMS) was chosen as the buoyant material in this study because of its many advantages: it is inexpensive, easy to produce, moldable, cures at low temperatures, and is both chemically and biologically inert enough for use in biomechanical devices [25]. More notably, PDMS is frequently used in the concentration of organic traces from aqueous domains through sorption in solid-phase extraction applications [26,27], and for separation and removal of crude oil from aqueous environments by adsorption [28-30]. This reported high adsorption affinity of PDMS could invigorate degradation by preconcentrating pollutants on its surface near the attached photocatalyst. PDMS has been previously used as a substrate for $\mathrm{TiO}_{2}$ in microfluidic reactors [12], and the two have been used to make hybrid films [31] that are useful as photocatalytic agents. There have also been successful studies in which $\mathrm{TiO}_{2}$ has been coupled with PDMS for self-cleaning, superhydrophobic surfaces, and coatings which exhibit removal of organic dyes under UV radiation $[15,32,33]$. However, the range of application of these materials for removal of harmful species in aqueous domains may be limited to environmentally-isolated reactors where buoyancy is not a necessity. Both $\mathrm{PDMS}$ and $\mathrm{TiO}_{2}$ are common components for materials used in biomedical devices [34,35], but no reports of the hybrid as a substrate in a discrete buoyant photocatalyst for water detoxification have been communicated to our knowledge.

We $[36,37]$ and others [38] have previously used microbubble fabrication techniques for PDMS to produce highly-porous, millimeter-scale beads. Drop-casting a cross-linking emulsion of PDMS dispersed in water into a heated water bath results in rapid curing of the PDMS into millimeter-scale beads. The microbubble form of PDMS within the emulsion is retained in the solid morphology, resulting in porous beads with a dense microstructure. These beads have high surface-area-to-volume (SAV) ratios, which is desirable in a photocatalytic substrate for maximizing photocatalyst exposure with minimal amounts of substrate to reduce total volume needed to accomplish the same removal properties. The porous PDMS bead substrate represents a completely new approach to buoyant photocatalyst materials. $\mathrm{TiO}_{2}$ was chosen in this study as a proof of concept because it provided the widest set of reports against which we could compare our new material. However, we emphasize that in principle any photocatalyst can be attached to PDMS beads through this simple fabrication method. The focus of this study is directed towards morphology and extent of $\mathrm{TiO}_{2}$ inoculation, kinetics of methylene blue (MB) removal, and reusability of these materials. Ultimately, we show that a high-functioning, discrete, buoyant photocatalyst using PDMS beads as the host material has been achieved.

\section{Results and Discussion}

\subsection{Physical Characterization}

The density of the beads, even those loaded with $\mathrm{TiO}_{2}$, is such that they float readily in aqueous solution, but sink in lower-density organic solvents, including $\mathrm{n}$-heptane, which has a density of $0.684 \mathrm{~g} / \mathrm{mL}$. For large-scale removal operations, this has the advantage of concentrating the photocatalyst at the interface between a low-density organic pollutant and the aqueous phase, thereby facilitating the photocatalytic degradation process.

\subsubsection{Material Morphology}

Scanning electron microscopy (SEM) images of beads created with and without $\mathrm{TiO}_{2}$ are compared in Figure 1. The pristine PDMS beads (Figure 1a) and ones inoculated with $\mathrm{TiO}_{2}$ (Figure 1b) show similar surface features, including convex morphologies, consistent with previous work where PDMS is dispersed in water and cured [36-38]. This provides evidence that $\mathrm{TiO}_{2}$ incorporation into PDMS by this method does not reduce the previously-reported high surface area of the host material [36]. Figure $1 \mathrm{c}, \mathrm{d}$ are close-up images of the same pristine $\mathrm{PDMS}$ and $\mathrm{TiO}_{2}$ inoculated beads, respectively, where rough surface features at the submicron level are observed. This roughness increases $\mathrm{TiO}_{2}$ exposure on the surface of the PDMS beads. It appears that the micromorphologies associated 
with emulsion formation for the pristine PDMS beads are slightly affected by inclusion of $\mathrm{TiO}_{2}$ into the fabrication process. This attachment is consistent with work by others who have successfully incorporated $\mathrm{TiO}_{2}$ into PDMS structures [15,32,33]. Washing of the beads in water, hexanes, and methanol does not remove appreciable amounts of $\mathrm{TiO}_{2}$ from the surface, nor does sonication, implying that the structures are robustly incorporated onto the polymer surface.
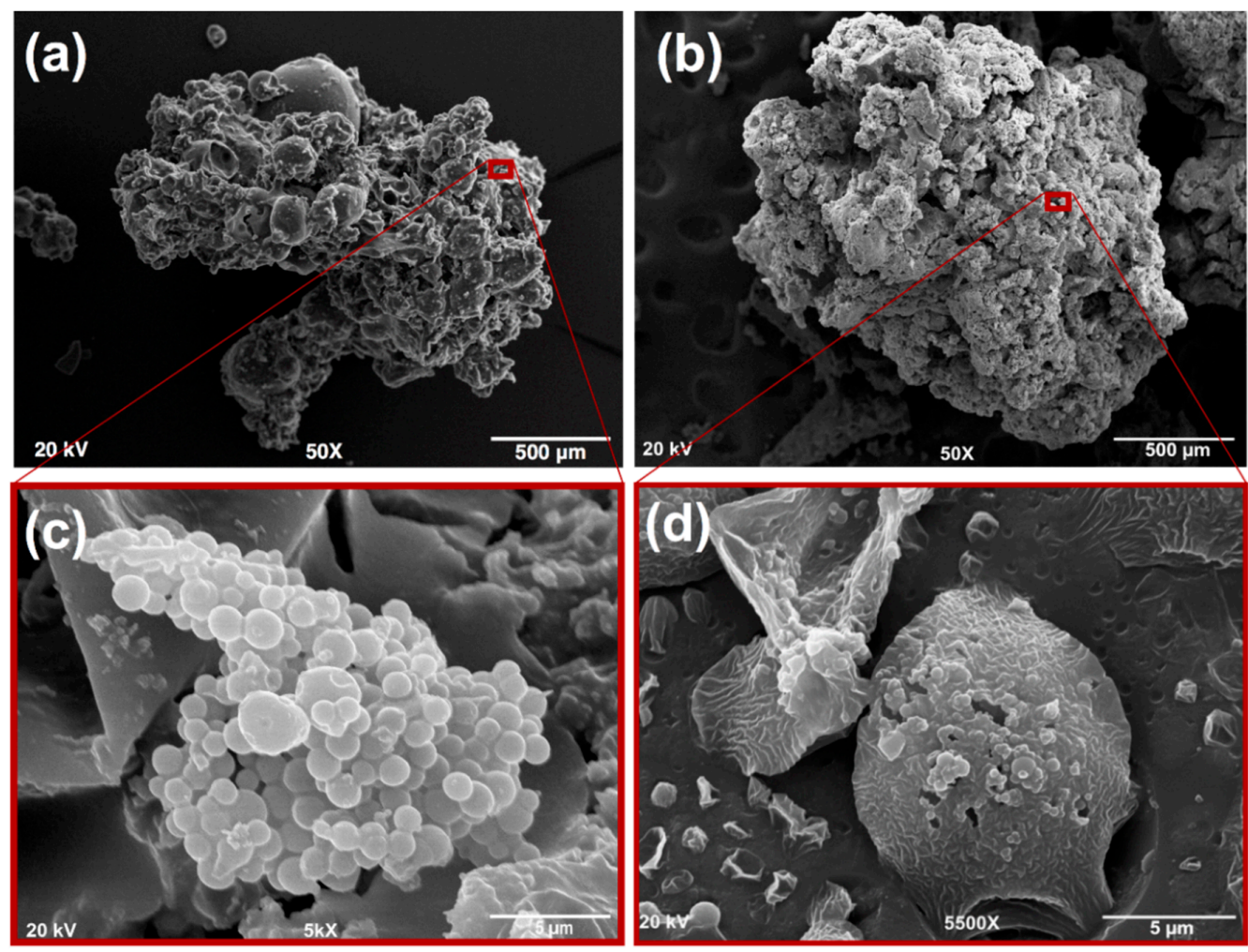

Figure 1. SEM images of representative poly(dimethylsiloxane) (PDMS) beads with (a) no $\mathrm{TiO}_{2}$ and (b) with a mass ratio of $20 \% \mathrm{TiO}_{2}$ added to the emulsion. Red rectangles indicate the areas in which images of the magnified regions for (c) the inert PDMS bead and (d) the $20 \% \mathrm{TiO}_{2} / \mathrm{PDMS}$ bead were measured. The convex porosity of the beads increases the surface area per bead, allowing a high density of available $\mathrm{TiO}_{2}$ for photocatalytic degradation.

Elemental analysis by energy-dispersive $X$-ray spectroscopy (EDS) (shown in the Supporting Information along with corresponding SEM images in Figure S1) was performed to assess $\mathrm{TiO}_{2}$ loads as summarized in Table 1. EDS Ti\% is the mass percent of Ti relative to Si found using EDS analysis (the spectra and corresponding images are in Supporting Information, Figure S1). Standard deviations $(\sigma)$ for EDS Ti\% were found by recording EDS spectra for three different beads for each respective load.

Table 1. Loading ratios determined by energy-dispersive X-ray spectroscopy (EDS). Mass percent ratios $\left(\mathrm{Mass} \% \mathrm{TiO}_{2}\right.$ ) refer to the mass of $\mathrm{TiO}_{2}$ added to the emulsion divided by the mass of the PDMS in the emulsion during synthesis.

\begin{tabular}{cc}
\hline Mass \%TiO $_{2}$ & EDS Ti $\% \pm \sigma$ \\
\hline 0 & 0 \\
5 & $6.6 \pm 0.8$ \\
10 & $11 \pm 1$ \\
20 & $15 \pm 3$ \\
\hline
\end{tabular}

The EDS data suggest that the loading of $\mathrm{TiO}_{2}$ at the surface is proportional to the amount of $\mathrm{TiO}_{2}$ suspended in solution. While washing the $20 \% \mathrm{TiO}_{2} / \mathrm{PDMS}$ beads directly after fabrication, the runoff 
appeared to be cloudy and white in color, characteristic of a $\mathrm{TiO}_{2}$ suspension. This did not occur for the $5 \%$ and $10 \% \mathrm{TiO}_{2} / \mathrm{PDMS}$ beads, providing evidence that a photocatalyst loading limit is reached using this method of host material fabrication. Adding $30 \%$ mass $\mathrm{TiO}_{2}$ to $\mathrm{PDMS}$ interfered with the stability of the PDMS microbubbles in water, preventing emulsion formation by the water bath sonicator.

\subsubsection{Surface Area}

Surface-area-to-volume measurements based on Brunauer-Emmett-Teller (BET) nitrogen isotherm analysis are summarized in Table 2. Compared to pristine beads, the $20 \%$ load beads show an increase in surface area per mass unit of sample. This is likely attributed to the increase in surface area due to the incorporation of $\mathrm{TiO}_{2}$ particles. This increase in surface area is consistent with the SEM images shown in Figure 1c,d, which show additional surface roughening by addition of crystalline $\mathrm{TiO}_{2}$ particles. A substantial increase in surface area was observed following three cycles of degradation. Because both the Raman and X-ray diffraction (XRD) characterizations suggest that the $\mathrm{TiO}_{2}$ remains intact and incorporated into the polymer surface after $\mathrm{MB}$ degradation, we interpret this increase in SAV to indicate some minor degradation of the PDMS microstructures into smaller pieces. In fact, it was noticed that a mass loss of $1-2 \%$ per trial started to occur in later consecutive trials. One area for future development of polymer-based supports will be to reduce susceptibility to such structural decay, but, as shown in the absolute photo-oxidation analysis below, this minor degradation does not appear to impact photocatalytic activity of these materials.

Table 2. Surface area (SA) per unit mass for pristine PDMS beads (from a previous paper [28]), anatase $\mathrm{TiO}_{2}$, and $20 \%-\mathrm{TiO}_{2} / \mathrm{PDMS}$. Addition of $\mathrm{TiO}_{2}$ to the beads increases the surface area of the bead. The increase compared to the pure $\mathrm{TiO}_{2}$ is an effect of the difference in density between $\mathrm{TiO}_{2}$ and polymer beads.

\begin{tabular}{cc}
\hline Sample & $\mathbf{S A ~}\left(\mathbf{m}^{\mathbf{2}} \mathbf{g}^{\mathbf{- 1}}\right)$ \\
\hline Pristine PDMS & 17.29 \\
$\mathrm{TiO}_{2}$ anatase (<21 nm) & 33.38 \\
$20 \% \mathrm{TiO}_{2} / \mathrm{PDMS}$ Before Degradation Trials $_{2}$ & 59.09 \\
$20 \% \mathrm{TiO}_{2} /$ PDMS After 3 Degradation Trials & 275.28 \\
\hline
\end{tabular}

\subsection{3. $\mathrm{TiO}_{2}$ Crystal Structures}

Raman spectra and XRD of the $\mathrm{TiO}_{2} / \mathrm{PDMS}$ beads were recorded to ensure that the method used to inoculate the PDMS beads and that the extensive exposure to UV radiation during degradation trials did not affect the anatase crystal structure. The Raman spectra of $\mathrm{TiO}_{2}$ only, $\mathrm{TiO}_{2} / \mathrm{PDMS}$ beads after synthesis, $\mathrm{TiO}_{2} / \mathrm{PDMS}$ beads after three degradation trials, and inert PDMS beads are depicted in Figure 2. Frequencies of the Raman bands observed for $\mathrm{TiO}_{2}$ are 398,515 , and $640 \mathrm{~cm}^{-1}$. The band at $398 \mathrm{~cm}^{-1}$ is reportedly assigned to the $B_{1 \mathrm{~g}}$ vibrational mode of the anatase crystal structure [39], The band at $515 \mathrm{~cm}^{-1}$ is reported to be a doublet of the $A_{1 \mathrm{~g}}$ and $B_{1 \mathrm{~g}}$ modes, while the band at $640 \mathrm{~cm}^{-1}$ is the degenerate $\mathrm{E}_{\mathrm{g}}$ vibrational mode of the anatase crystal structure.

The Raman spectra of $\mathrm{TiO}_{2} / \mathrm{PDMS}$ beads before and after the degradation trials exhibit slight differences relative to the spectrum of $\mathrm{TiO}_{2}$ alone. The band at $515 \mathrm{~cm}^{-1}$ appears to broaden slightly, and a new band emerges just above $700 \mathrm{~cm}^{-1}$. To investigate this, the Raman spectrum of the solid PDMS beads was recorded as a reference. It appears that both the additional peak and the peak broadening observed for both $\mathrm{TiO}_{2}$ /PDMS spectra are due to the solid structure of PDMS. If rutile phase $\mathrm{TiO}_{2}$ were present, bands would be expected to appear at $447 \mathrm{~cm}^{-1}, 612 \mathrm{~cm}^{-1}$, and $827 \mathrm{~cm}^{-1}$ for the $E_{g}, A_{1 g}$, and $B_{2 g}$ Raman active vibrational modes, respectively. Ultimately, the Raman spectra of $\mathrm{TiO}_{2} / \mathrm{PDMS}$ beads before and after the degradation trials are nearly identical and possess all bands observed for $\mathrm{TiO}_{2}$ anatase, which indicates that neither the synthesis method, nor the degradation trials, alter the anatase crystal structure of $\mathrm{TiO}_{2}$. This conclusion is further supported by the XRD spectra shown in Figure S2 of the Supporting Information, which show peaks consistent with anatase 
$\mathrm{TiO}_{2}$ only, along with broad features due to the (noncrystalline) polymer substrate. The broader features in the XRD are consistent with those typically found for nanofine $\mathrm{TiO}_{2}$ powders.

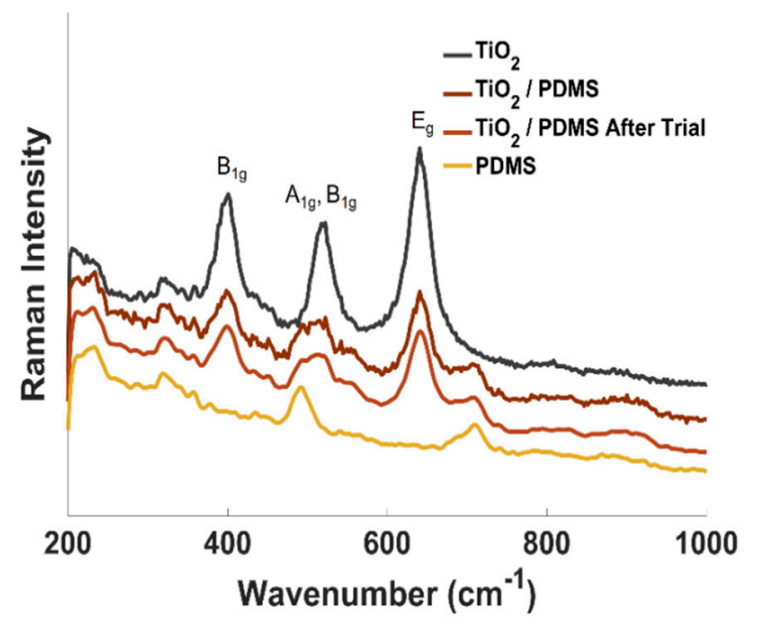

Figure 2. Raman spectrum of pure anatase $\mathrm{TiO}_{2}, \mathrm{TiO}_{2} / \mathrm{PDMS}$ after synthesis, $\mathrm{TiO}_{2} / \mathrm{PDMS}$ after three consecutive 3-h long degradation trials, and inert PDMS beads.

\subsection{Degradation of Methylene Blue}

Photocatalytic activity of the beads was assessed by photocatalytic degradation of methylene blue (MB), a commonly used standard for such experiments. Typical visible absorption spectra as a function of exposure time in the presence of $5 \% \mathrm{TiO}_{2} / \mathrm{PDMS}$ beads are shown in Figure 3. During the course of the experiment, both peaks in the 550 to $750 \mathrm{~nm}$ range decay exponentially with time. Total peak area in this range (by integrating the peaks over the absorption range) is used to assess the kinetics. The inset of Figure 3 shows a plot of the natural log of the peak area relative to the peak area of MB solution prior to addition of photocatalytic beads. The strong linearity of this plot is consistent with the expected first-order kinetics in the degradation of MB: the negative slope for each experiment indicates the rate constant, $k_{T O T}$, for the removal of $\mathrm{MB}$ from solution. Representative natural-log plots as a function of time are shown in Figure 4, with results summarized in Table 3. It is clear from the plot that $k_{\mathrm{TOT}}$ increases with $\mathrm{TiO}_{2}$ load, as expected. $\mathrm{All} \mathrm{TiO}_{2} / \mathrm{PDMS}$ beads performed substantially better at removal of MB from solution than inert PDMS or by direct photolysis of MB without any additives. For trials containing $20 \%$ and $10 \% \mathrm{TiO}_{2} / \mathrm{PDMS}$ beads, the slope deviates from linearity at longer times, indicating Langmuir kinetics (previously observed in MB removal by adsorption onto $\mathrm{TiO}_{2}$ ) [40] resulting from an equilibrium between the aqueous and adsorbed species. This decreases and eventually ceases the rate of MB removal by adsorption, but does not affect the rate constant for photocatalysis, as we explain with our kinetic model below. In this work, adsorption equilibrium was not attempted prior to illumination under UV light, thus the decrease in the removal rate of $\mathrm{MB}$ observed near the end of the trials is attributed to approaching adsorption equilibrium of $\mathrm{MB}$ onto the $\mathrm{TiO}_{2}$ anchored to the PDMS. Therefore, the value of $k_{T O T}$ for trials with $20 \%$ and $10 \%$ loads was elucidated from the first linear region of the natural $\log$ plots in Figure 4 . This means that at least part of the removal represented by $k_{T O T}$ is due to adsorption of $\mathrm{MB}$ from the solution phase to the $\mathrm{TiO}_{2}$ surface. An additional linear fit was applied to the second linear region for the higher loads and the negative slope calculated to elucidate another rate constant, $k_{T O T e q}$. For the $10 \%$ and $20 \%$ load beads, $k_{T O T e q}$ is clearly consistent with the sum of $k_{h v}^{\prime}$ and $k_{P O}^{\prime}$, but could not be determined for the $5 \%$ load beads due to the lack of a clear, second linear region. Both linear fits are shown in the Supporting Information and Figure S3; results are summarized in Table 3. Table 3 also includes data from off-substrate photocatalytic degradation experiments in which an amount of $\mathrm{TiO}_{2}$ equivalent to that present on the $\mathrm{TiO}_{2} / \mathrm{PDMS}$ beads with $20 \%$ load was suspended directly in the MB solution. Kinetics measured in those experiments are used to compare the rate constant for MB removal in 
the presence of a direct $\mathrm{TiO}_{2}$ suspension and the buoyant photocatalyst presented here. The value of $k_{T O T}$ for the $20 \%$ loaded beads is only an order of magnitude less than the recorded rate constant of direct suspension. This is significant considering the available photocatalytic surface area in the direct suspension is far greater than the $\mathrm{TiO}_{2}$ trapped by PDMS beads (and thus, the chances for $\mathrm{MB}$ degradation): the volume over which the $\mathrm{MB}$ is distributed is more than ten times the volume of the beads in the buoyant case, which should mean that the rate is reduced by more than an order of magnitude. This surprising advantage is perhaps due to the high surface-area of the PDMS beads and their ability to preconcentrate the pollutant at the active photocatalyst surface.

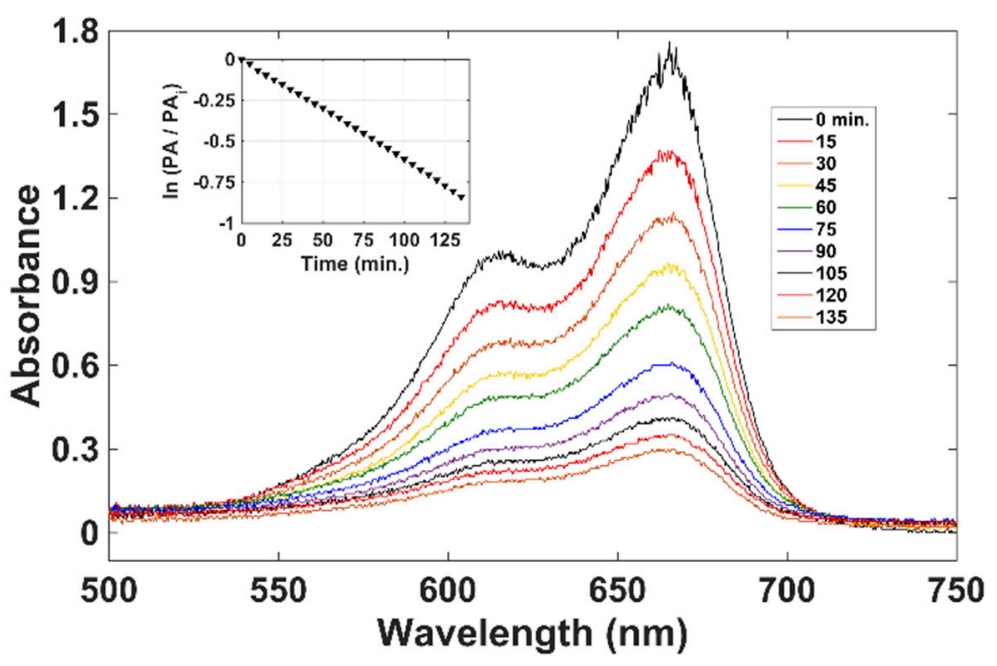

Figure 3. Visible absorption spectra of methylene blue (MB) solution during irradiation in the presence of photocatalytic beads, showing loss of MB from solution as a function of time. Inset plot shows the natural log of the peak area ratio as a function of time suggesting the removal rate of $\mathrm{MB}$ is first-order. The beads used are made with a $5 \% \mathrm{TiO}_{2}: \mathrm{PDMS}$ mass ratio in the emulsion.

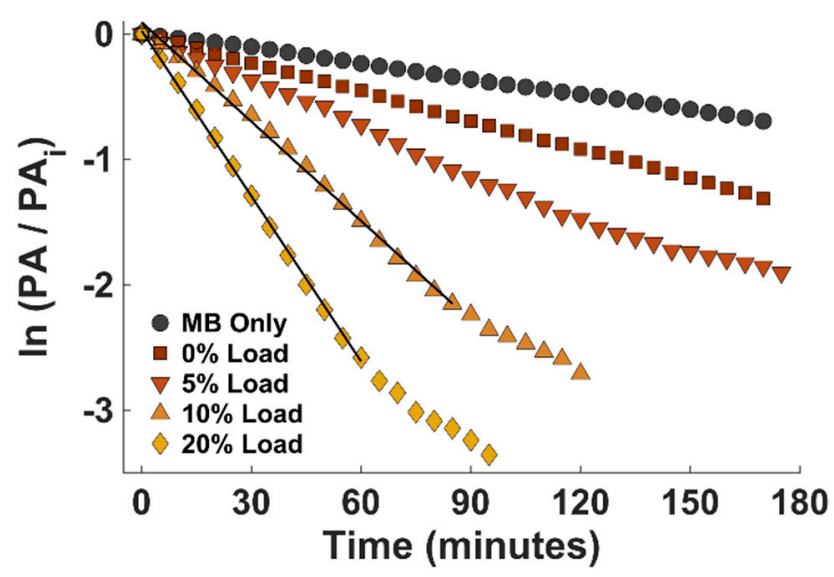

Figure 4. First-order kinetic plots (natural log of concentration relative to initial concentration) for MB solution using a series of beads with different $\mathrm{TiO}_{2}$ loads. Also shown are inert PDMS beads ( $0 \%$ load) and photolysis of MB solution without any additives (MB Only). The slopes of the lines indicate the rate constant for loss of $\mathrm{MB}$ by adsorption, photolysis, and photocatalytic degradation. Two linear regions are observed for $20 \%$ and $10 \%$ loads due to $\mathrm{MB}$ adsorption equilibrium onto the beads resulting in a slight decrease in the removal rate; the value of $k_{T O T}$ is based on the first region, indicated with a line in the plot. 
Table 3. Photocatalytic degradation rate constants for different beads produced in the study. The pseudo-first order rate constants for adsorption $\left(k_{a d s}^{\prime}\right)$ and photolysis $\left(k_{h v}^{\prime}\right)$, elucidated by control experiments, contribute to the total removal of $\mathrm{MB}\left(k_{T O T}\right)$; the fraction attributed to nonadsorption is assigned $k_{T O T e q}$. The pseudo-first order rate constant $k_{P O}^{\prime}$ represents $\mathrm{MB}$ removal only by photocatalytic degradation which is found by using the kinetic model developed in this work. Errors listed for rate constants are standard deviations, calculated from three trials.

\begin{tabular}{cccccc}
\hline Mass $\% \mathrm{TiO}_{\mathbf{2}}$ & $\boldsymbol{k}_{\text {TOT }}\left(\mathbf{m i n}^{-\mathbf{1}}\right)$ & $\boldsymbol{k}_{\text {TOTeq }}\left(\mathbf{m i n}^{-\mathbf{1}}\right)$ & $\boldsymbol{k}_{\boldsymbol{h v}}^{\prime}\left(\mathbf{m i n}^{-\mathbf{1}}\right)$ & $\boldsymbol{k}_{\text {ads }}^{\prime}\left(\mathbf{m i n}^{-\mathbf{1}}\right)$ & $\boldsymbol{k}_{\mathbf{P O}}^{\prime}\left(\mathbf{m i n}^{-\mathbf{1}}\right)$ \\
\hline 0 & - & - & $0.0045 \pm 0.0003$ & - & - \\
5 & $0.008 \pm 0.003$ & - & $0.0045 \pm 0.0003$ & $0.0015 \pm 0.0006$ & $0.002 \pm 0.003$ \\
10 & $0.032 \pm 0.003$ & $0.013 \pm 0.002$ & $0.0045 \pm 0.0003$ & $0.018 \pm 0.002$ & $0.009 \pm 0.004$ \\
20 & $0.042 \pm 0.002$ & $0.017 \pm 0.001$ & $0.0045 \pm 0.0003$ & $0.026 \pm 0.001$ & $0.012 \pm 0.002$ \\
$\left(\mathrm{TiO}_{2}\right.$ Suspension $)$ & $0.36 \pm 0.03$ & - & - & - & - \\
\hline
\end{tabular}

A comparison of the $\mathrm{TiO}_{2} / \mathrm{PDMS}$ beads presented here was made to other materials presented in the literature that used a polymer-based photocatalyst support in the degradation of MB. The PDMS beads shown here outperform polystyrene sheets (per gram, per $\mathrm{W}$ of radiation) inoculated with $\mathrm{TiO}_{2}$ [23] likely because of the increase in SAV ratio. However, the materials described in this work do not outperform waste polystyrene beads inoculated with $\mathrm{ZnO}$ [41].

It is known that $\mathrm{ZnO}$ outperforms $\mathrm{TiO}_{2}$ in the degradation of $\mathrm{MB}$ [42] because it has a higher quantum efficiency for the degradation of organic pollutants [43]. However, $\mathrm{ZnO}$ has been reported to be susceptible to photo-induced corrosion under acidic conditions, hindering its reliability for commercial or large-scale applications [44]. Nonetheless, this underscores the need for the incorporation of other photocatalysts into the porous PDMS substrates described in this work; such work is already underway in our laboratory. Our materials have several major advantages over previously reported buoyant photocatalysts based on polymer substrates. First, as indicated by the BET results, microbubble-fabricated PDMS is far more porous than standard polymer morphologies. Second, the beads are small and rotate freely in water, but are not completely hydrophobic, which is essential for photocatalytic degradation applications. Finally, the methods we describe are highly scalable, and could be used to produce large quantities in preparation for disastrous environmental discharge.

\subsection{Absolute Photocatalytic Degradation Rates}

Because most authors report only $k_{T O T}$, we have compared our results with this value to assess the relative value of our materials. However, the well-known adsorption of $\mathrm{MB}$ and other similar compounds to $\mathrm{TiO}_{2}$ complicates measurements of photocatalytic degradation. The ability of the $\mathrm{TiO}_{2}$-innoculated beads to adsorb $\mathrm{MB}$ from solution increases as the actual load of $\mathrm{TiO}_{2}$ on the beads increases. Most previously published photocatalysis results are unable to separate effects of direct photolysis and adsorption to the catalyst from true photocatalytic oxidation. Separation of these effects is particularly challenging if experiments are performed in a turbid suspension of analyte and photocatalyst unless those experiments also account for the opacity of the suspension. However, the photocatalyst is not dispersed. Because we monitor only the degradation of $\mathrm{MB}$, we use a kinetic model to disentangle the contributions of adsorption $\left(k_{a d s}\right)$ and direct photolysis $\left(k_{h v}\right)$ from genuine photocatalytic degradation $\left(k_{P O}\right)$ by oxidizers in solution, collectively $\left\{O_{x}\right\}$ :

$$
\begin{gathered}
\mathrm{MB}+\mathrm{TiO}_{2} \stackrel{k_{\text {ads }}}{\longrightarrow}{\mathrm{MB} @ \mathrm{TiO}_{2}} \\
\mathrm{MB}+h v \stackrel{k_{h v}}{\longrightarrow}\{\text { Products }\}_{h v} \\
\mathrm{MB}+\{\mathrm{O} x\} \stackrel{k_{P}}{\longrightarrow}\{\text { Products }\}_{o x}
\end{gathered}
$$


In our mechanism, each of the paths to removal of $\mathrm{MB}$ from solution (our experimental observable) is first order in $\mathrm{MB}$, and, given the very low concentrations of $\mathrm{MB}$ in solution, pseudo-first order overall, allowing the total reaction to be considered to have a single, first-order rate constant, $k_{T O T}$, which is the sum of the pseudo-first order rate constants for the three component reactions:

$$
k_{T O T}=k_{a d s}\left[\mathrm{TiO}_{2}\right]+k_{h v}[h v]+k_{P O}[O x]=k_{a d s}^{\prime}+k_{h v}^{\prime}+k_{P O}^{\prime}
$$

Thus, calculation of $k_{P O}[O x]$, which is a measure of actual photocatalytic activity, without the contributions from other aspects, is possible by determination of the values of $k_{a d s}\left[\mathrm{TiO}_{2}\right]$ and $k_{h v}[h v]$ and subtraction from total removal rate constant, $k_{T O T}$. The results for the pseudo-first-order rate constants are summarized in Table 3, with some additional analysis in the Supporting Information.

To demonstrate the importance of adsorption of $\mathrm{MB}$ to $\mathrm{TiO}_{2}$ and elucidate $k_{a d s}^{\prime}$, total absorbance measurements of $\mathrm{MB}$ solution are measured as a function of time following the addition of $\mathrm{TiO}_{2}$ beads (with and without photocatalyst) to the solution while stirring, but in a dark environment. Adsorption curves are shown in Figure 5. Like the anticipated degradation kinetics, adsorption appears to follow first-order kinetics. Adsorption contributes a non-negligible (but not dominant) route to the removal of MB from solution and should be considered when evaluating the true photocatalytic activity of any photocatalyst material. Because our materials allow us to separate these effects, we do so, but it should be noted that, in terms of application for environmental cleaning, $k_{T O T}$ may be the single most important gauge of usefulness.

We also note that, while adsorption complicates measurement of MB photocatalytic degradation, beads removed from the solution following complete degradation of the MB available in solution are white or light blue in color, while those recovered from the solution after the dark experiments were stained a deep blue. This indicates that degradation was occurring (and likely on or near the bead) rather than only adsorption, in a period of approximately $50 \mathrm{~min}$ for the heavier loads of $\mathrm{TiO}_{2}$. Thus, while adsorption contributes slightly to the rate of $\mathrm{MB}$ removal, it may also enhance the actual degradation of $\mathrm{MB}$ by preconcentration of the analyte on the surface of the photocatalyst. Thus, the final $k_{P O}^{\prime}$ values listed in Table 3 are minimum values; the actual photo-oxidation rate constant may be even higher.

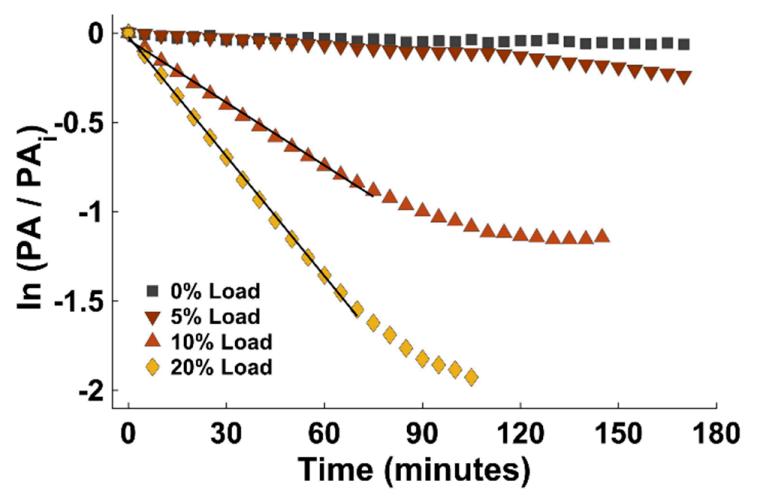

Figure 5. Adsorption curves for $\mathrm{MB}$ to $\mathrm{TiO}_{2} / \mathrm{PDMS}$ beads. Visible absorbance spectra are collected in the absence of radiation capable of photolysis or photocatalytic activation to isolate the extent to which $\mathrm{MB}$ adsorbs to the beads as a function of time. Due to adsorption equilibrium, removal rates of MB for $10 \%$ and $20 \%$ loads began depreciating during the trial. Thus, the negative slope of the linear regions (indicated in the plot) are reported as $k_{a d s}$.

In all cases, the rate constants during irradiation are at least twice as high as in the dark experiments. This suggests that, although some removal of MB from solution is due to adsorption directly onto the bead surface, a significant amount of photocatalytic degradation is also occurring. The extent of MB removed by direct photolysis, $k_{h v}^{\prime}$, was measured by performing experiments with inert PDMS on the surface of the MB solution $\left(0 \% \mathrm{TiO}_{2} / \mathrm{PDMS}\right.$ load). This rate constant acted as a lower 
limit to MB removal in the presence of PDMS beads. Since these beads contained no photocatalyst, degradation of $\mathrm{MB}$ by photooxidative species is impossible. Also, because $\mathrm{TiO}_{2}$ (not PDMS) is directly responsible for $\mathrm{MB}$ adsorption, photolysis must be the only phenomenon responsible for $\mathrm{MB}$ removal when inert PDMS beads are placed in the reaction vessel and subjected to degradation trials. Thus, $k^{\prime} h v$ solely represents the rate constant for the photolysis of MB in the presence of inert PDMS beads.

After removal contributions from direct photolysis and adsorption are considered, the photooxidative removal rate constant, $k_{P O}^{\prime}$, accounts for a significant portion of the overall removal of $\mathrm{MB}$. The rate constant for the degradation of $\mathrm{MB}$ in a direct suspension of $\mathrm{TiO}_{2}$ (using the same equivalent mass of photocatalyst loaded onto the PDMS beads) was determined for comparison. The experimental design for testing MB degradation uses top-irradiation, meaning that opacity of the beads would result in an expected lowering of the removal rate compared to MB solution without any beads, so this is a lower limit for removal by photocatalytic degradation. The direct suspension rate constant was found to be just over an order of magnitude higher than the recorded $k_{P O}^{\prime}$. At first consideration, this may seem low, but it is not, given that the photocatalyst is restricted to the surface, meaning far less volume is accessible at any time. Instead, this comparison indicates that, although anchoring the photocatalyst to the polymer substrate reduces its available surface area, the porous microstructure and the high SAV ratio of the PDMS beads provides an appreciable amount of exposure of the photocatalyst to the aqueous environment, but without the substantial environmental drawbacks of a suspended photocatalyst.

\subsection{Robustness of Materials}

The beads retain photocatalytic activity when reused multiple times. Consecutive cycles of MB degradation trials were performed under the same experimental conditions, using beads that had been subjected to a degradation period after recovering by gravity filtration and washing with DI-water and hexanes prior to drying at room temperature. Figure 6 shows $k_{P O}^{\prime}$ for the same photocatalytic beads with varying $\mathrm{TiO}_{2}$ load, each subjected to five consecutive degradation trials of $\mathrm{MB}$. A slight increase in the rate constant was observed for the second trials for each $\mathrm{TiO}_{2} / \mathrm{PDMS}$ load. This is attributed to minor contributions of adsorption of $\mathrm{MB}$ onto the photocatalyst. Although adsorption equilibrium is met within the first trial, washing the beads in various solvents prior to resubmitting them to degradation trials removes small amounts of adsorbed MB. Although $k_{P O}^{\prime}$ for the third trial of the $20 \%$ load drops below that of the $10 \%$ load, the experimental uncertainties suggest that this is within expected measurement fluctuations. Thus, while adsorption is improved by increasing load beyond $10 \%$, the absolute photocatalytic activity may saturate at $10 \%$ instead due to photon saturation of the trapped photocatalysts. Further consecutive trials display only minor fluctuations in $k_{P O}^{\prime}$, indicating that the reusability of the $\mathrm{TiO}_{2} / \mathrm{PDMS}$ beads as a buoyant photocatalyst is strong.

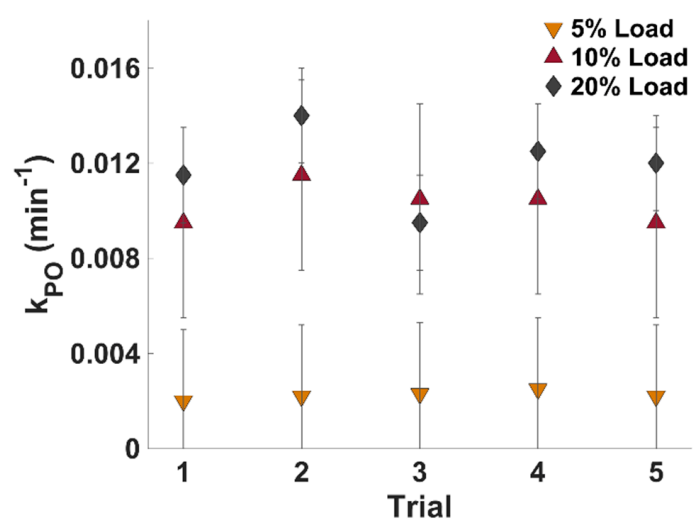

Figure 6. Reuse data for photocatalytic $\mathrm{TiO}_{2} / \mathrm{PDMS}$ beads, showing $k_{\mathrm{TOT}}$ for three consecutive runs of $3 \mathrm{~h}$ each for several different loads of $\mathrm{TiO}_{2}$. The error bars represent the uncertainty for each value, as reported in Table 3. 


\section{Materials and Methods}

\subsection{Fabrication and Morphology Analysis}

The microbubble fabrication methods used to synthesize porous PDMS beads have been detailed elsewhere [36,37]. Briefly, vinyl-terminated PDMS (Aldrich, St. Louis, MO, USA) is dissolved in $n$-heptane (Aldrich, St. Louis, MO, USA), and is then emulsified with an aqueous surfactant solution (sodium dodecyl sulfate, Aldirch, St. Louis, MO, USA) by sonication. Triethoxysilane (Gelest, Morrisville, PA, USA) is added as a cross linker and, after a brief cross-linking period, the emulsion is heat cured by dropwise addition into a $75{ }^{\circ} \mathrm{C}$ water bath to form 1-2 mm beads with convex micrometer-scale surface porosity. To incorporate photocatalyst into the beads, $\mathrm{TiO}_{2}$ was added to approximately $3 \mathrm{~mL}$ of the emulsion mixture prior to sonication. To incorporate $\mathrm{TiO}_{2}$ photocatalyst into the PDMS beads, $0.05,0.1$, and $0.2 \mathrm{~g}$ of $\mathrm{TiO}_{2}$ (anatase, nanofine, Aldrich, St. Louis, MO, USA) was added to the emulsion, corresponding to $5 \%, 10 \%$, and $20 \%$ photocatalyst load with respect to the initial weight of PDMS. After curing, beads are dried at room temperature and rinsed with hexanes, then with water, then finally dried at room temperature prior to analysis or photocatalytic testing.

\subsection{Physical Characterization}

PDMS beads, anatase $\mathrm{TiO}_{2}$, and beads inoculated with $\mathrm{TiO}_{2}$ were all characterized using an array of standard analytical techniques. Surface morphologies were imaged using a scanning electron microscope (SEM, JSM-6510LV, JEOL, Tokyo, Japan) at an accelerating voltage of $20 \mathrm{kV}$. Beads were subjected to Au sputter coating (SC500, EMSCOPE, Barcelona, Spain) prior to SEM analysis to obtain clear images of the bead's surface. Energy dispersive spectroscopy (EDS, 550i, iXRF Systems, Austin, TX, USA) was used to provide a rough estimate of the $\mathrm{TiO}_{2}$ coverage for each load. Surface area measurements per gram of sample for pristine beads and for the $20 \% \mathrm{TiO}_{2}$ load beads were measured using an ASAP 2420 Brunauer-Emmett-Teller (BET), nitrogen isotherm analyzer (Micrometrics, Norcross, GA, USA) to ensure that the addition of $\mathrm{TiO}_{2}$ did not damage the high $\mathrm{SAV}$ of the samples. Samples of $20 \% \mathrm{TiO}_{2}$ beads were also measured following three dye removal cycles to ensure bead and photocatalyst integrity during the course of the photocatalytic process. All BET samples were first dried and degassed in a vacuum oven at $120^{\circ} \mathrm{C}$ for $6 \mathrm{~h}$. Raman scattering and powder X-ray diffraction (SmartLab, Rigaku, Tokyo, Japan) to determine that the $\mathrm{TiO}_{2}$ remained in the photocatalytically active anatase phase throughout the synthetic process. A back-scatter Raman spectrometer (Desktop L-Peakseeker, Agiltron, Woburn, MA, USA) with a spectral range of 200 to $3000 \mathrm{~cm}^{-1}$ was also used as a fast, non-invasive technique to detect the presence of $\mathrm{TiO}_{2}$ and to ensure its anatase crystal structure within PDMS beads before and after degradation experiments remained intact. Solid samples of $\mathrm{TiO}_{2}$, $\mathrm{PDMS}, \mathrm{TiO}_{2} / \mathrm{PDMS}$, and $\mathrm{TiO}_{2} / \mathrm{PDMS}$ after one degradation trial were packed into the corner of clean, unused plastic bags prior to Raman measurement with a probe designed for solid measurement; no signal is observed from the bag.

\subsection{Dye Removal Performance}

Photocatalytic activity was evaluated by degradation of aqueous MB solution stirred slowly with a magnetic stir bar under UV-visible light irradiation (unfiltered $300 \mathrm{~W}$ Xe-arc lamp, $40 \mathrm{~mW} / \mathrm{m}^{2}$ at the sample, Newport, Irvine, CA, USA). The lamp has substantial (but not constant photon flux over the wavelength range of 250 to $2500 \mathrm{~nm}$. The reaction was carried out in a 50-mL quartz round-bottom flask using $0.3 \mathrm{~g}$ of $\mathrm{TiO}_{2} / \mathrm{PDMS}$ beads floating in $20 \mathrm{~mL}$ of $30-\mu \mathrm{M}$ MB solution, which was cycled

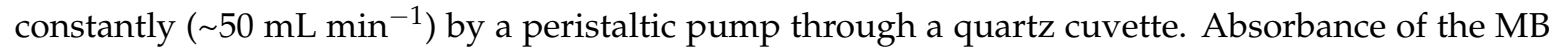
solution as a function of time was measured in the cuvette every 5 min over a period of $3 \mathrm{~h}$. Control experiments were performed using a MB solution alone to assess the rate of MB photolysis. Inert PDMS beads were also added to an MB solution as a control experiment to assess the extent of the effect that PDMS bead opacity has on the direct photolysis of MB. In an effort to disentangle the phenomena responsible for $\mathrm{MB}$ removal, additional control experiments were performed in the dark to measure $\mathrm{MB}$ 
adsorption onto PDMS beads (both with and without $\mathrm{TiO}_{2}$ ) without UV light exposure. For comparison to measurements using a dispersed photocatalyst, $0.06 \mathrm{~g} \mathrm{TiO}_{2}$ (equivalent to the amount present in $0.3 \mathrm{~g}$ of $20 \% \mathrm{TiO}_{2} / \mathrm{PDMS}$ beads) was suspended in $20 \mathrm{~mL}$ of $30-\mu \mathrm{M} \mathrm{MB}$ solution and exposed to the Xe-arc lamp. The suspension was slowly stirred with a magnetic stir-bar and wavelength-dependent reflectance measurements of the solution were recorded to assess the photocatalytic degradation of MB. To confirm the validity of the reflectance measurements, similar trials were performed using inert PDMS beads instead of $\mathrm{TiO}_{2}$. The resulting rate constants of $\mathrm{MB}$ degradation were within uncertainties of the values reported for absorbance measurements, confirming wavelength-dependent reflectance measurements are a sound method of comparison.

\section{Conclusions}

We have demonstrated the fabrication of a PDMS-supported $\mathrm{TiO}_{2}$ photocatalyst that is both buoyant and discrete. Due to their complex micromorphology and porous structure, the PDMS beads permit significant activation of the anchored photocatalyst and possess strong reusability. A relatively low load of photocatalyst is required to accomplish high quality degradation of MB from solution, in part because the PDMS substrate microstructure exposes a significant portion of the anchored photocatalyst, allowing good access to both water and analyte, a necessary feature for effective buoyant photocatalysts, and one not accomplished by less structured morphologies. This is of particular interest for large-scale removal of pollutants that are poorly soluble in water. The bead morphology also has the advantage that it does little to inhibit direct photolysis by UV radiation, a problem which can arise for both suspensions of free photocatalysts or, presumably, non-UV-transparent polymer sheets. All of these features make this particular design a leading candidate for management of acute environmental disasters such as petroleum or industrial chemical discharges into large bodies of water.

Optimization of the bead surface morphology to increase SAV will further develop this new approach to buoyant photocatalysts. As we have demonstrated previously [37], the addition of $\mathrm{ZnCl}_{2}$ to the aqueous phase of the emulsion results in more concave sites on the cured polymer beads, which could provide an increased surface load of $\mathrm{TiO}_{2}$. We have begun to explore this and other morphological effects to optimize the processes and photocatalytic activities identified here based on this initial report. Another important expansion of this work will be the incorporation of more promising photocatalysts to the surface. Incorporation of other photocatalysts, including $\mathrm{ZnO}$, sensitized $\mathrm{TiO}_{2}$ derivatives, and other metal oxides, will allow more direct comparison with polystyrene beads described by other authors [41]. It is possible that the siloxane structure of PDMS are particularly amenable to the incorporation of metal oxides, and may therefore also serve as a buoyant substrate for metal organic framework photocatalysts, particularly those with a high oxygen content, or oxometallates, which have shown substantial progress in recent years. The results reported here are the first steps in a new direction for environmental cleaning, one we hope will substantially improve our ability to respond to otherwise catastrophic pollution events at major water resources.

Supplementary Materials: The following are available online at http:/ / www.mdpi.com/2073-4344/8/10/482/s1, Figure S1. Energy dispersive spectra and corresponding surface SEM images for 5\% (a,b) 10\% (c,d) and 20\% (e,f) $\mathrm{TiO}_{2} /$ PDMS loads used to assess the extent of photocatalyst load onto the buoyant substrate, Figure S2. XRD data for (bottom to top) pure PDMS beads, pure anatase nanopowder, and $20 \% \mathrm{TiO}_{2}$ loaded PDMS beads, before and after three cycles of MB degradation, Figure S3. First and second linear fits of natural log of the peak area ratio for $\mathrm{MB}$ absorption. The negative slope of the first linear fit represents the rate constant $k_{T O T}$, while the second represents the rate constant $k_{T O T E q}$.

Author Contributions: The manuscript was written through contributions of both authors. Both authors have given approval to the final version of the manuscript. Conceptualization, J.R.B. and M.J.N.; Data curation, J.R.B.; Formal analysis, J.R.B.; Funding acquisition, M.J.N.; Investigation J.R.B.; Methodology, J.R.B. and M.J.N.; Project administration, M.J.N.; Resources, M.J.N.; Software, J.R.B.; Supervision, M.J.N.; Validation, J.R.B. and M.J.N.; Visualization, J.R.B.; Writing—original draft, J.R.B.; Writing—review \& editing, M.J.N.

Funding: This work has been supported in part by the Western Kentucky University Office of Research and Office of the Provost through a Faculty-Undergraduate Student Engagement (FUSE) grant.

Conflicts of Interest: The authors declare no conflict of interest. 


\section{References}

1. Okamoto, K.-I.; Yamamoto, Y.; Tanaka, H.; Itaya, A. Kinetics of heterogeneous photocatalytic decomposition of phenol over anatase $\mathrm{TiO}_{2}$ powder. Bull. Chem. Soc. Jpn. 1985, 58, 2023-2028. [CrossRef]

2. Zahraa, O.; Sauvanaud, L.; Hamard, G.; Bouchy, M. Kinetics of atrazine degradation by photocatalytic process in aqueous solution. Int. J. Photoenergy 2003, 5, 87-93. [CrossRef]

3. Nosaka, Y.; Nosaka, A.Y. Generation and Detection of Reactive Oxygen Species in Photocatalysis. Chem. Rev. 2017, 117, 11302-11336. [CrossRef] [PubMed]

4. Tachikawa, T.; Fujitsuka, M.; Majima, T. Mechanistic Insight into the $\mathrm{TiO}_{2}$ Photocatalytic Reactions: Design of New Photocatalysts. J. Phys. Chem. C 2007, 111, 5259-5275. [CrossRef]

5. Lazar, M.; Varghese, S.; Nair, S. Photocatalytic Water Treatment by Titanium Dioxide: Recent Updates. Catalysts 2012, 2, 572-601. [CrossRef]

6. Pelizzetti, E.; Minero, C. Mechanism of the photo-oxidative degradation of organic pollutants over $\mathrm{TiO}_{2}$ particles. Electrochim. Acta 1993, 38, 47-55. [CrossRef]

7. Bekbölet, M.; Lindner, M.; Weichgrebe, D.; Bahnemann, D.W. Photocatalytic detoxification with the thin-film fixed-bed reactor (TFFBR): Clean-up of highly polluted landfill effluents using a novel $\mathrm{TiO}_{2}$-photocatalyst. Sol. Energy 1996, 56, 455-469. [CrossRef]

8. Mehrjouei, M.; Müller, S.; Möller, D. Design and characterization of a multi-phase annular falling-film reactor for water treatment using advanced oxidation processes. J. Environ. Manag. 2013, 120, 68-74. [CrossRef] [PubMed]

9. Kühn, K.P.; Chaberny, I.F.; Massholder, K.; Stickler, M.; Benz, V.W.; Sonntag, H.-G.; Erdinger, L. Disinfection of surfaces by photocatalytic oxidation with titanium dioxide and UVA light. Chemosphere 2003, 53, 71-77. [CrossRef]

10. Rtimi, S.; Pulgarin, C.; Sanjines, R.; Kiwi, J. Kinetics and mechanism for transparent polyethylene-TiO $\mathrm{F}_{2}$ films mediated self-cleaning leading to MB dye discoloration under sunlight irradiation. Appl. Catal. B 2015, 162, 236-244. [CrossRef]

11. Paz, Y.; Luo, Z.; Rabenberg, L.; Heller, A. Photooxidative self-cleaning transparent titanium dioxide films on glass. J. Mater. Res. 2011, 10, 2842-2848. [CrossRef]

12. Lamberti, A. Microfluidic photocatalytic device exploiting $\mathrm{PDMS} / \mathrm{TiO}_{2}$ nanocomposite. Appl. Surf. Sci. 2015, 335, 50-54. [CrossRef]

13. Fujishima, A.; Zhang, X.; Tryk, D.A. $\mathrm{TiO}_{2}$ photocatalysis and related surface phenomena. Surf. Sci. Rep. 2008, 63, 515-582. [CrossRef]

14. Kumar, S.G.; Devi, L.G. Review on Modified $\mathrm{TiO}_{2}$ Photocatalysis under UV/Visible Light: Selected Results and Related Mechanisms on Interfacial Charge Carrier Transfer Dynamics. J. Phys. Chem. A 2011, 115, 13211-13241. [CrossRef] [PubMed]

15. Tavares, M.T.S.; Santos, A.S.F.; Santos, I.M.G.; Silva, M.R.S.; Bomio, M.R.D.; Longo, E.; Paskocimas, C.A.; Motta, F.V. $\mathrm{TiO}_{2} / \mathrm{PDMS}$ nanocomposites for use on self-cleaning surfaces. Surf. Coat. Technol. 2014, 239, 16-19. [CrossRef]

16. Jin, L.; $\mathrm{Wu}, \mathrm{H} . ;$ Morbidelli, M. Synthesis of Water-Based Dispersions of Polymer/ $\mathrm{TiO}_{2} \mathrm{Hybrid}_{\text {Nanospheres. }}$ Nanomaterials 2015, 5, 1454-1468. [CrossRef] [PubMed]

17. Di Credico, B.; Redaelli, M.; Bellardita, M.; Calamante, M.; Cepek, C.; Cobani, E.; D’Arienzo, M.; Evangelisti, C.; Marelli, M.; Moret, M.; et al. Step-by-Step Growth of HKUST-1 on Functionalized $\mathrm{TiO}_{2}$ Surface: An Efficient Material for $\mathrm{CO}_{2}$ Capture and Solar Photoreduction. Catalysts 2018, 8, 353. [CrossRef]

18. Wan, W.; Yu, S.; Dong, F.; Zhang, Q.; Zhou, Y. Efficient $\mathrm{C}_{3} \mathrm{~N}_{4}$ /graphene oxide macroscopic aerogel visible-light photocatalyst. J. Mater. Chem. A 2016, 4, 7823-7829. [CrossRef]

19. Liu, W.; Cai, J.; Li, Z. Self-Assembly of Semiconductor Nanoparticles/Reduced Graphene Oxide (RGO) Composite Aerogels for Enhanced Photocatalytic Performance and Facile Recycling in Aqueous Photocatalysis. ACS Sustain. Chem. Eng. 2015, 3, 277-282. [CrossRef]

20. Singh, S.; Mahalingam, H.; Singh, P.K. Polymer-supported titanium dioxide photocatalysts for environmental remediation: A review. Appl. Catal. A 2013, 462, 178-195. [CrossRef] 
21. Zhang, L.; Xing, Z.; Zhang, H.; Li, Z.; Zhang, X.; Zhang, Y.; Li, L.; Zhou, W. Multifunctional Floating Titania-Coated Macro/Mesoporous Photocatalyst for Efficient Contaminant Removal. ChemPlusChem 2015, 80, 623-629. [CrossRef]

22. Singh, S.; Singh, P.K.; Mahalingam, H. Novel floating $\mathrm{Ag}^{+}$-doped $\mathrm{TiO}_{2} /$ polystyrene photocatalysts for the treatment of dye wastewater. Ind. Eng. Chem. Res. 2014, 53, 16332-16340. [CrossRef]

23. Singh, S.; Singh, P.; Mahalingam, H. An Effective and Low-Cost $\mathrm{TiO}_{2} /$ Polystyrene Floating Photocatalyst for Environmental Remediation. Int. J. Environ. Res. 2015, 9, 535-544.

24. Tu, W.; Lin, Y.-P.; Bai, R. Removal of phenol in aqueous solutions by novel buoyant Composite photocatalysts and the kinetics. Sep. Purif. Technol. 2013, 115, 180-189. [CrossRef]

25. Encyclopedia of Materials: Science and Technology, 2nd ed.; Wiley: Hoboken, NJ, USA, 2001; pp. $2480-2482$.

26. Vrana, B.; Popp, P.; Paschke, A.; Schüürmann, G. Membrane-enclosed sorptive coating. An integrative passive sampler for monitoring organic contaminants in water. Anal. Chem. 2001, 73, 5191-5200. [CrossRef] [PubMed]

27. Kim, T.-Y.; Alhooshani, K.; Kabir, A.; Fries, D.P.; Malik, A. High pH-resistant, surface-bonded sol-gel titania hybrid organic-inorganic coating for effective on-line hyphenation of capillary microextraction (in-tube solid-phase microextraction) with high-performance liquid chromatography. J. Chromatogr. A 2004, 1047, 165-174. [CrossRef]

28. Zhang, A.; Chen, M.; Du, C.; Guo, H.; Bai, H.; Li, L. Poly(dimethylsiloxane) oil absorbent with a three-dimensionally interconnected porous structure and swellable skeleton. ACS Appl. Mater. Interfaces 2013, 5, 10201-10206. [CrossRef] [PubMed]

29. Li, N.; Li, T.; Lei, X.; Fu, B.; Liao, W.; Qiu, J. Preparation and characterization of porous PDMS beads for oil and organic solvent sorption. Polym. Eng. Sci. 2014, 54, 2965-2969. [CrossRef]

30. Park, I.; Efimenko, K.; Sjöblom, J.; Genzer, J. Rapid removal of organics and oil spills from waters using silicone rubber "sponges". J. Dispers. Sci. Technol. 2009, 30, 318-327. [CrossRef]

31. Iketani, K.; Sun, R.-D.; Toki, M.; Hirota, K.; Yamaguchi, O. Sol-gel-derived $\mathrm{TiO}_{2}$ /poly(dimethylsiloxane) hybrid films and their photocatalytic activities. J. Phys. Chem. Sol. 2003, 64, 507-513. [CrossRef]

32. Zhao, Y.; Liu, Y.; Xu, Q.; Barahman, M.; Lyons, A.M. Catalytic, Self-Cleaning Surface with Stable Superhydrophobic Properties: Printed Polydimethylsiloxane (PDMS) Arrays Embedded with $\mathrm{TiO}_{2}$ Nanoparticles. ACS Appl. Mater. Interfaces 2015, 7, 2632-2640. [CrossRef] [PubMed]

33. Silva, V.; Paschoalino, M.; Gonçalves, M.; Felisberti, M.; Jardim, W.; Yoshida, I. Silicone rubbers filled with $\mathrm{TiO}_{2}$ : Characterization and photocatalytic activity. Mater. Chem. Phys. 2009, 113, 395-400. [CrossRef]

34. Chen, Q.; Miyata, N.; Kokubo, T.; Nakamura, T. Bioactivity and mechanical properties of PDMS-modified $\mathrm{CaO}-\mathrm{SiO}_{2}-\mathrm{TiO}_{2}$ hybrids prepared by sol-gel process. J. Biomed. Mater. Res. 2000, 51, 605-611. [CrossRef]

35. Chen, Q.; Miyata, N.; Kokubo, T.; Nakamura, T. Effect of heat treatment on bioactivity and mechanical properties of PDMS-modified $\mathrm{CaO}-\mathrm{SiO}_{2}-\mathrm{TiO}_{2}$ hybrids via sol-gel process. J. Mater. Sci. Mater. Med. 2001, 12, 515-522. [CrossRef] [PubMed]

36. Salkic, S.; Eckler, L.H.; Nee, M.J. Non-invasive monitoring of photocatalytic degradation of x-ray contrast media using Raman spectrometry. J. Raman Spectrosc. 2013, 44, 1746-1752. [CrossRef]

37. Bertram, J.R.; Nee, M.J. Microbubble Fabrication of Concave-porosity PDMS Beads. J. Vis. Exp. 2015, e53440. [CrossRef] [PubMed]

38. Dufaud, O.; Favre, E.; Sadtler, V. Porous elastomeric beads from crosslinked emulsions. J. Appl. Polym. Sci. 2002, 83, 967-971. [CrossRef]

39. Balachandran, U.; Eror, N. Raman spectra of titanium dioxide. J. Solid State Chem. 1982, 42, $276-282$. [CrossRef]

40. Houas, A.; Lachheb, H.; Ksibi, M.; Elaloui, E.; Guillard, C.; Herrmann, J.-M. Photocatalytic degradation pathway of methylene blue in water. Appl. Catal. B 2001, 31, 145-157. [CrossRef]

41. Altın, İ; Sökmen, M. Buoyant photocatalyst based on $\mathrm{ZnO}$ immobilized on polystyrene beads for pollutants treatment. CLEAN Soil Air Water 2015, 43, 1025-1030. [CrossRef]

42. Fatin, S.; Lim, H.; Tan, W.; Huang, N. Comparison of photocatalytic activity and cyclic voltammetry of zinc oxide and titanium dioxide nanoparticles toward degradation of methylene blue. Int. J. Electrochem. Sci. 2012, 7, 9074-9084. 
43. Sakthivel, S.; Neppolian, B.; Shankar, M.; Arabindoo, B.; Palanichamy, M.; Murugesan, V. Solar photocatalytic degradation of azo dye: Comparison of photocatalytic efficiency of $\mathrm{ZnO}$ and $\mathrm{TiO}_{2}$. Sol. Energy Mater. Sol. Cells 2003, 77, 65-82. [CrossRef]

44. Fouad, O.; Ismail, A.; Zaki, Z.; Mohamed, R. Zinc Oxide Thin Films Prepared by Thermal Evaporation Deposition and Its Photocatalytic Activity. Appl. Catal. B 2006, 62, 144-149. [CrossRef] 